\title{
Revised screening scale to predict survival after insertion of a left ventricular assist device
}

\author{
Vivek Rao, MD, $\mathrm{PhD}$ \\ Mehmet C. Oz, MD \\ Margaret A. Flannery, FNP \\ Katharine A. Catanese, RN \\ Michael Argenziano, MD \\ Yoshifumi Naka, MD, PhD
}

From the Division of Cardiothoracic Surgery, New York Presbyterian Hospital and Columbia University, New York, NY.

Received for publication Sept 24, 2001; revisions requested Jan 3, 2002; revisions received July 15,2002 ; accepted for publication July 23, 2002.

Address for reprints: Vivek Rao, MD, $\mathrm{PhD}$, FRCS(C), Toronto General Hospital, 200 Elizabeth St, EN 14-222, Toronto, Ontario, M5G 2C4 Canada (E-mail: vivek.rao@uhn.on.ca).

J Thorac Cardiovasc Surg 2003;125:855-62

Copyright (C) 2003 by The American Association for Thoracic Surgery

$0022-5223 / 2003 \$ 30.00+0$

doi:10.1067/mtc.2003.111
Background: We previously calculated a risk factor summation score that successfully predicted survival after insertion of a left ventricular assist device. We sought to validate our previous score by using a single center's clinical experience and to determine emerging risk factors for mortality after device insertion.

Methods: The clinical records of 130 consecutive patients who received the HeartMate VE left ventricular assist device (Thoratec Corp, Pleasanton, Calif) at our institution between June 1996 and March 2001 as a bridge to transplantation were reviewed. Univariate and multivariable analyses were performed to determine the predictors of operative mortality after device insertion. Using the relative risks for each identified variable, we devised a new risk factor summation score. The new and old scores were then compared by using linear regression analyses to determine whether the revised score improved statistical accuracy.

Results: Overall operative mortality was $25 \%(\mathrm{n}=33)$. The old score successfully predicted operative mortality in the current patient population (operative mortality of $38 \%$ for score $>5$ vs $13 \%$ for score $\leq 5$ ). However, the revised score improved risk discrimination (operative mortality of $46 \%$ for a score $>5$ vs $12 \%$ for a score $\leq$ to 5). Statistical accuracy was comparable between scores, but the relationship between observed and predicted outcomes was improved with the revised score.

Conclusions: The changing demographic profile and management of patients presenting for mechanical circulatory support has led to a change in the predictors of mortality after device insertion. Periodic remodeling and recalibration of risk indices helps to accurately predict outcomes in high-risk patient groups and identifies emerging risk factors for mortality.

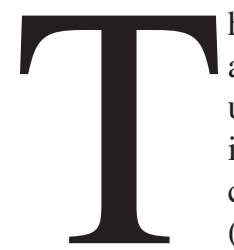

he successful use of mechanical circulatory assistance for the management of end-stage heart disease has resulted in the widespread use of a variety of devices approved by the Food and Drug Administration as bridges to cardiac transplantation. ${ }^{1-3}$ For the 2 most common long-term implantable left ventricular assist devices (LVADs), the vented electric HeartMate (Thoratec Corp, Pleasanton, Calif) and the Novacor (WorldHeart Corp, Ottawa, Ontario, Canada), survival to transplantation is greater than $70 \% .^{2-4}$

We have previously reported a preoperative screening scale that predicts operative mortality in patients receiving the 1000IP or 1205VE HeartMate LVAD. ${ }^{5}$ In developing this scale, we reviewed 56 consecutive patients who received a device either at our institution or at The Cleveland Clinic Foundation. All patients met the hemodynamic criteria for LVAD insertion proposed by Norman and colleagues ${ }^{6}$ : a cardiac index of less than $2.0 \mathrm{~L} \cdot \mathrm{min}^{-1} \cdot \mathrm{m}^{-2}$ with a left atrial or pulmonary 
TABLE 1. Previous risk factor summation score

\begin{tabular}{lccc}
\hline Variable & Relative risk & P value & Weighting \\
\hline $\mathrm{U} / 0<30 \mathrm{~mL} / \mathrm{h}$ & 3.9 & .002 & 3 \\
CVP $>16 \mathrm{~mm} \mathrm{Hg}$ & 3.1 & .02 & 2 \\
Ventilated & 2.9 & .04 & 2 \\
PT $>16 \mathrm{~s}$ & 2.4 & .08 & 2 \\
Redo surgery & 1.7 & .21 & 1 \\
WBC $>15,000 / \mathrm{mm}^{3}$ & 1.1 & .86 & 0 \\
Temperature $>101.5^{\circ} \mathrm{F}$ & 0.0 & .56 & 0
\end{tabular}

$U / O$, Urine output; $C V P$, central venous pressure; $P T$, prothrombin time; $W B C$, white blood cell count.

capillary wedge pressure of greater than $20 \mathrm{~mm} \mathrm{Hg}$. A 10 -point scale was developed by using 5 factors found to be statistically significant by means of univariate analysis (Table 1). Each factor was weighted according to its relative risk, and a summation score of greater than 5 was found to be associated with a prohibitive risk of death (67\% operative mortality).

In 1999, we published the results of an LVAD bridgeto-transplant network to manage patients with postcardiotomy shock. ${ }^{7}$ In this high-risk subgroup of patients, we reported a survival to hospital discharge of $66 \%$ compared with a reported survival of less than $20 \%$ in patients supported with triple-inotropic therapy and intra-aortic balloon pump counterpulsation. ${ }^{8}$ Since the publication of that report, we have noticed, in our institution, a substantial increase in the proportion of patients presenting for LVAD insertion with a temporary device in situ (bridge-to-bridge support). These patients have often undergone 2 previous sternotomies with cardiopulmonary bypass and present for a third bypass run in a span of less than 48 hours for insertion of a long-term device as a bridge to transplantation. Not surprisingly, these patients pose a challenge for perioperative management and have severe abnormalities of hepatic, renal, and pulmonary function.

Because of the changing nature of our patient population, we believed that a reassessment of our screening scale was appropriate. We now have the statistical power to develop a score on the basis of multivariable analyses of data obtained from a single institution. As suggested by Ivanov and colleagues, ${ }^{9}$ periodic recalibration of existing indices and remodeling of screening scores are necessary to ensure that emerging risk factors are not inadvertently overlooked. The advantage of a single-center study is the uniformity of management practices, which removes this variable from the outcome measures in multicenter analyses. However, an admitted limitation is the potential lack of generalizability. Therefore, the purpose of this report is to validate the previous multicenter screening scale in a cohort of 130 consecutive patients receiving LVADs at our institution and to determine whether there are emerging risk factors that predict mortality in this challenging patient population.
TABLE 2. Perioperative demographic characteristics

\begin{tabular}{lccc}
\hline Variable & $\begin{array}{c}\text { Survivors } \\
(\mathbf{n}=\mathbf{9 7})\end{array}$ & $\begin{array}{c}\text { Nonsurvivors } \\
(\mathbf{n}=\mathbf{3 3})\end{array}$ & $\boldsymbol{P}$ value \\
\hline Age (y) & $49 \pm 14$ & $52 \pm 14$ & .2 \\
Female gender & $17(18)$ & $8(24)$ & .4 \\
Diagnosis & & & .03 \\
$\quad$ CAD & $45(46)$ & $24(73)$ & \\
DCM & $40(41)$ & $6(18)$ & \\
$\quad$ Other & $12(12)$ & $3(9)$ & \\
Pre-LVAD & $11(11)$ & $15(45)$ & $<.001$ \\
Pre-RVAD & $3(3)$ & $7(21)$ & $<.001$ \\
Acute MI & $25(26)$ & $15(45)$ & .03 \\
Postcardiotomy & $18(19)$ & $20(61)$ & $<.001$ \\
U/O < 30 mL/hr & $62(64)$ & $23(70)$ & .6 \\
CVP $>16 \mathrm{~mm} \mathrm{Hg}$ & $57(59)$ & $26(79)$ & .04 \\
PT > 16 s & $48(49)$ & $24(73)$ & .02 \\
Ventilated & $38(38)$ & $28(85)$ & $<.001$ \\
CVVHD & $29(30)$ & $24(73)$ & $<.001$ \\
Redo surgery & $15(15)$ & $6(18)$ & .7 \\
\hline
\end{tabular}

Numbers in parentheses refer to the corresponding percentage frequency. $C A D$, Ischemic cardiomyopathy; $D C M$, dilated cardiomyopathy; Pre-LVAD, temporary LVAD in situ at the time of HeartMate insertion; Pre-RVAD, temporary RVAD in situ at the time of HeartMate insertion; $M I$, myocardial infarction; $U / O$, urine output; $C V P$, central venous pressure; $P T$, prothrombin time; $C V V H D$, perioperative need for continuous venovenous hemodialysis.

\section{Methods}

\section{Data Sources}

All patients receiving the single-lead VE HeartMate LVAD were observed by a dedicated nursing and medical staff. Pertinent perioperative clinical and demographic data were entered prospectively into an institutional database. One hundred thirty consecutive patients who received a device between June 1996 and March 2001 were included in this study. Details of surgical technique and perioperative management have been published previously. ${ }^{1,10}$

\section{Statistical Analysis}

The SAS statistical software program version 8 for Windows (SAS Institute, Cary, NC) was used for all statistical analyses. Categoric data are expressed as their absolute or percentage frequency values and were compared with $\chi^{2}$ or Fisher exact tests where appropriate. Continuous data are expressed as the means $\pm \mathrm{SD}$ and were compared by using the Student $t$ test.

\section{Model Derivation}

The original summation score (Table 1) was derived from a univariate analysis of 56 patients who received an LVAD as a bridge to transplant at Columbia Presbyterian Hospital or The Cleveland Clinic Foundation. ${ }^{5}$ We validated this index by using it in our current data set, which contains no patient overlap with the previous study. A stepwise logistic regression model was developed by using the 5 criteria specified in the original scoring system, and the regression coefficients were then entered into an equation to yield the predicted probability of mortality for each patient. Patients were then grouped according to risk score (0-10), 
and the mean probability of mortality was calculated for each score.

The revised score was developed by reviewing all explanatory variables with a univariate $P$ value of less than .25 for operative mortality after LVAD insertion. A revised score was then calculated by using the variables selected in a multivariable model, with weights assigned according to their odds ratios (ORs), univariate relative risks, or both (Table 2). Once again, a stepwise logistic regression model was developed by using the 5 criteria specified in the new summation score, and the probability of mortality was calculated for each score as above.

The previous (old score) and revised (new score) summation scales were then evaluated by plotting the predicted versus observed mortalities at each risk score and calculating the slope and $y$-intercept of the weighted linear regression line. A slope of 1 and $y$-intercept of 0 indicates a perfect fit of predicted value to observed outcomes and suggests an accurate summation score. The different scoring scales were then compared by analyzing differences in slopes and intercepts by using analysis of covariance. In addition, model discrimination was assessed by using the area under the receiver operating characteristic (ROC) curve for each score. ${ }^{9}$

\section{Results}

The demographic characteristics of the study population are presented in Table 2, with a comparison of survivors and nonsurvivors. Most of the previously identified risk factors continue to be associated with higher mortality, with the exception of renal insufficiency as defined by poor urine output. Redo surgery (defined as remote sternotomy) no longer predicts death. New univariate predictors of mortality include previous LVAD or right ventricular assist device (RVAD) placement (bridge-to-bridge support), postcardiotomy shock, acute myocardial infarction, and ischemic cardiomyopathy. A stepwise linear regression model identified a ventilated patient (OR, 5.7; 95\% confidence interval, 1.8-17.5) and a previous LVAD (OR, 2.8; 95\% confidence interval, 1.0-7.9) as independent predictors of mortality after device insertion. Table 3 shows the risk factors used to devise the revised summation score and the relative risk ratios for the variables excluded from the new score.

Figure 1 demonstrates the operative mortality associated with both the old summation score and the new revised score in the current patient population. Consistent with our previous report, scores of greater than 5 in the old scale were associated with significantly higher operative mortality $(38 \%$ vs $13 \%, P=.001)$. In the new scale a score of greater than 5 continued to be associated with higher mortality ( $46 \%$ vs $12 \%, P<.001$ ); however, the discrimination between high and low risk was improved. Figure 2 compares the discrimination between high and low risk on the basis of scores of greater than 5 in both the old and new scales. The sensitivity of a score of greater than 5 with the new scale was similar to that of the old scale $(73 \%, P=.8)$. However, the specificity of a score of less than 6 was $71 \%$
TABLE 3. Revised risk factor summation score

\begin{tabular}{lcccc}
\hline Variable & Sample size & Relative risk & $\boldsymbol{P}$ value & Weighting \\
\hline Ventilated & 66 & 5.3 & $<.0001$ & 4 \\
Postcardiotomy & 38 & 3.3 & $<.0001$ & 2 \\
Pre-LVAD & 26 & 3.3 & $<.0001$ & 2 \\
CVP $>16 \mathrm{~mm} \mathrm{Hg}$ & 83 & 2.1 & .04 & 1 \\
PT $>16 \mathrm{~s}$ & 72 & 2.1 & .02 & 1 \\
\hline Variables excluded & Sample size & Relative risk & Univariate $\boldsymbol{P}$ value \\
\hline Pre-RVAD & 10 & 3.2 & \multicolumn{2}{c}{.0007} \\
Diagnosis CAD & 69 & 2.0 & \multicolumn{2}{c}{.03} \\
Acute MI & 40 & 1.7 & \multicolumn{2}{c}{.03} \\
U/O <30 mL/h & 85 & 1.2 & \multicolumn{2}{c}{.55} \\
Redo surgery & 21 & 1.2 & \multicolumn{2}{c}{.71} \\
\end{tabular}

$C V P$, Central venous pressure; $P T$, prothrombin time; $C A D$, ischemic cardiomyopathy; $M I$, myocardial infarction; $U / 0$, urine output.

by using the new scale and only $60 \%$ with the old scale $(P=.2)$.

Figure 3 displays the ROC curves associated with both the old and new scoring systems. Both scores demonstrated adequate statistical precision to predict operative mortality, although the new scale was associated with a slightly higher area under the ROC curve. Figure 4 plots the observed versus predicted mortality for all 130 patients by using both the old scale (upper panel) and the new scale (lower panel), along with a linear regression line constructed for each scale. The old scale yielded excellent correlation between predicted and observed values $(r=0.88)$; however, the slope of the relation was significantly different from 1 ( $\beta=$ $1.46 \pm 0.05, P<.01)$, and the y-intercept was significantly different from $0(\mathrm{c}=-11.7 \pm 1.4, P<.001)$, suggesting that the old scale significantly underestimated actual mortality in higher-risk patients. In contrast, the new scale demonstrated a similar correlation $(r=0.87)$, with a slope closer to $1(\beta=1.00 \pm 0.04, P=.8)$ and a $y$-intercept not different from $0(\mathrm{c}=0.004 \pm 1.3, P=.9)$.

\section{Discussion}

Chronic congestive heart failure is the only cardiovascular diagnosis increasing in prevalence. ${ }^{11}$ As the use of mechanical assistance to support this population increases, the indications for device implantation need to be clarified. Although individual patients will undoubtedly benefit from this technology, the cost-benefit ratio of this therapy might not justify device insertion in all potential recipients. ${ }^{12}$ The increased interest in public reporting of surgical results has led to the development of many risk indices for mortality after isolated coronary bypass surgery. ${ }^{9,13-15}$ Unfortunately, the relatively small clinical experience in single centers has made derivation of similar risk indices for LVAD insertion difficult.

To provide a common denominator with which to begin 


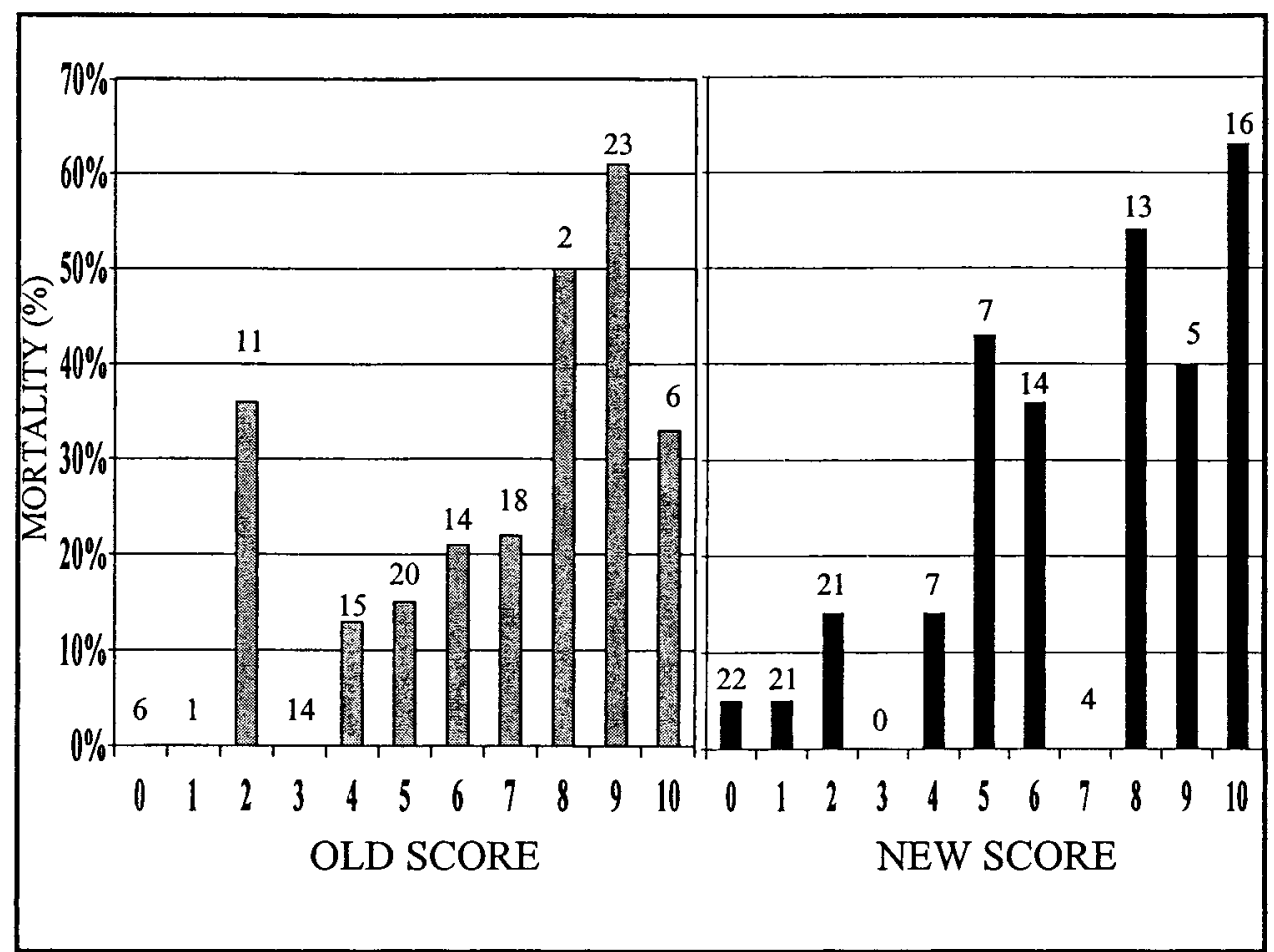

Figure 1. Relationship between operative mortality and risk factor summation score. Both the old score and the new score successfully predicted risk after LVAD insertion. In both scales a score of greater than $\mathbf{5}$ is associated with high mortality.

discussions with referring physicians, we devised a risk factor summation scale that successfully predicted operative mortality in HeartMate recipients. ${ }^{5}$ To achieve the necessary statistical power, we combined the clinical experience of Columbia Presbyterian Hospital and The Cleveland Clinic Foundation to analyze a total of 56 LVAD recipients. The summation score derived from this earlier study performed satisfactorily in the present analysis, which included a single center's experience with 130 consecutive patients.

There are advantages and disadvantages of deriving a risk index from a single center's experience. The major advantages include uniformity of care and relevance to that center's patient population. The disadvantage lies in its potential lack of generalizability to larger patient populations and to centers with different management algorithms. Regardless of the data sources, it is useful to periodically update and remodel existing risk indices to detect emerging hazards and to assess the relative effect of known risk factors.

The purpose of the original scale was to provide LVAD teams with a quick screening tool to determine operative risk in potential device recipients. At our center, we found this tool to be extremely useful when initiating dialogues with referring physicians from "spoke" centers (ie, referral centers without the capability of providing long-term me- chanical assistance or transplantation). ${ }^{7}$ However, our clinical observation that the increasing proportion of patients presenting with postcardiotomy shock faced increased operative risk prompted us to reexamine our risk factor summation score. Our analysis provided some expected results but also revealed surprising trends in previously important variables.

\section{Effect of Postcardiotomy Shock}

The 3 most important variables in the new scoring scale all reflect the high-risk nature of a patient with postcardiotomy cardiogenic shock. Most series report a survival to hospital discharge of only $20 \%$ to $40 \% .7,8,16,17$ A ventilated patient presenting with a temporary LVAD in situ has already achieved a score of 8 (predicted mortality of 37\%). The fact that mechanical ventilation emerged as the most important multivariable predictor of death $(\mathrm{OR}, 5.7)$ reflects the underlying status of a patient who is deemed to require endotracheal intubation. Although measurements of inspired oxygen concentration, oxygen saturation, or $\mathrm{Po}_{2}$ might provide valuable clinical information, the deliberate act of endotracheal intubation is a binomial criterion with high discriminatory powers. As a result of these considerations, mechanical ventilation earned 4 points on the revised scoring scale 


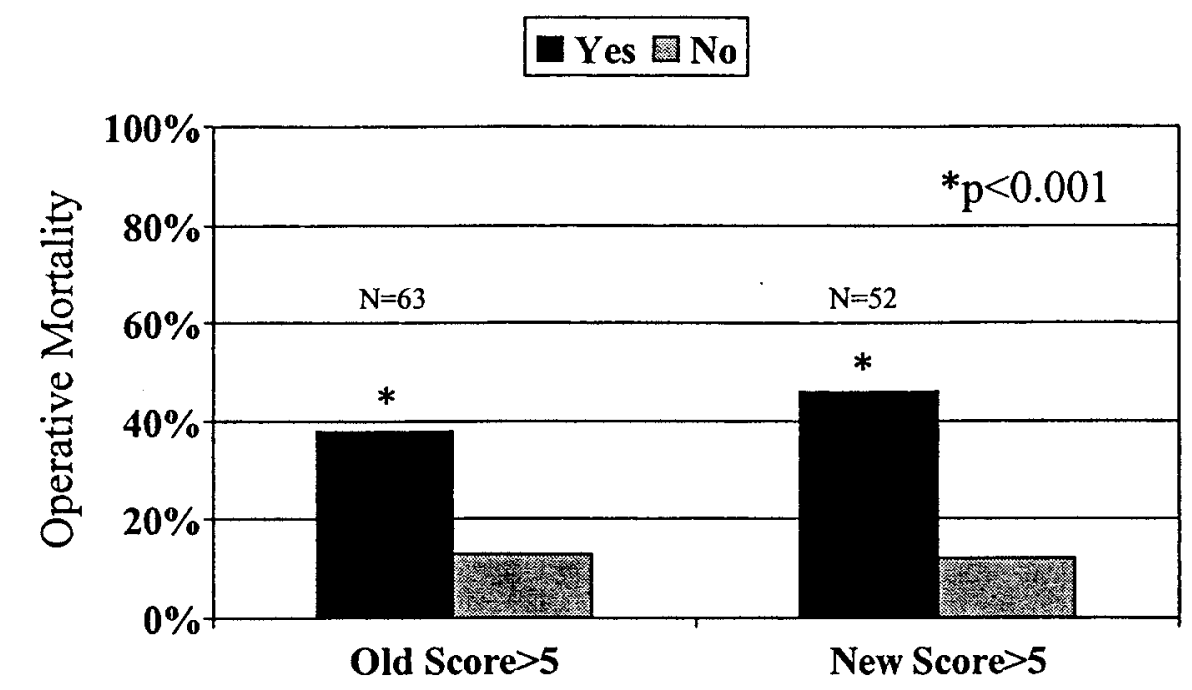

\section{Preoperative Risk Factor}

Figure 2. Discrimination between high and low risk (score $>5$ ) for mortality after LVAD insertion. Although both scales successfully predicted risk, the new scale improved discrimination.

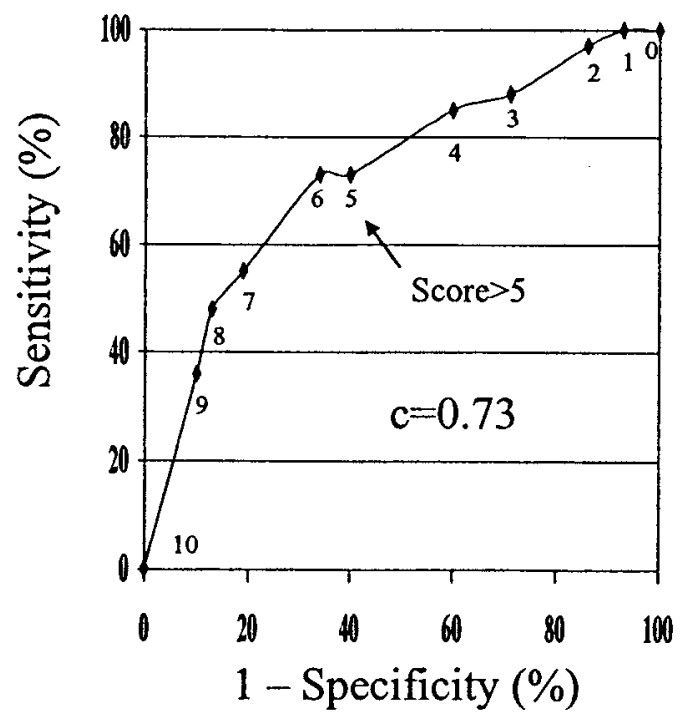

OLD Score

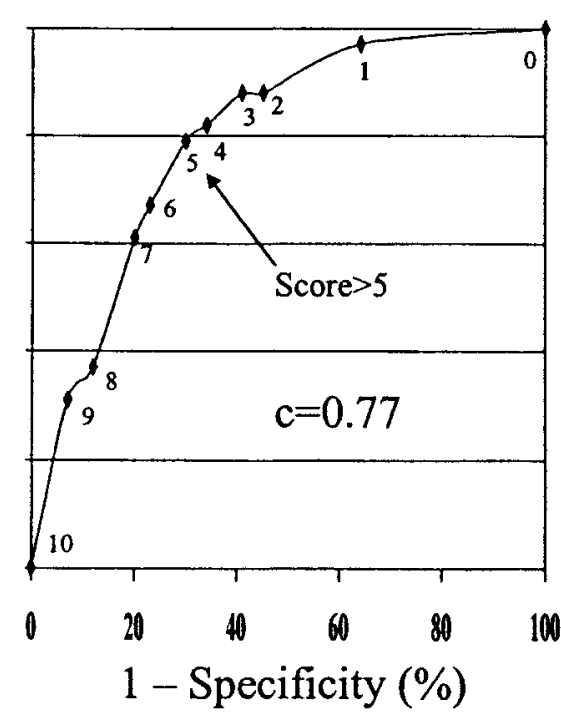

NEW Score

Figure 3. ROC curves for the old (left) and new (right) risk factor summation scores. Both scores display adequate statistical accuracy, with slightly better accuracy in the new score (area under the ROC curve, $\mathrm{c}=0.77$ vs 0.73 ).

and represents the single most important risk factor for mortality after LVAD insertion.

The technical aspects of redo surgery, including the increased risk of bleeding and myocardial injury during sternal reentry, are no longer statistically significant, with a relative risk of only 1.2 compared with 1.7 in the old scale. Postcardiotomy shock conferred a relative risk of 3.3 and replaced redo surgery as a variable in the new scale. Most patients with a temporary LVAD suffered from postcardiotomy shock. All but 4 patients (1 device failure, 1 epi- 


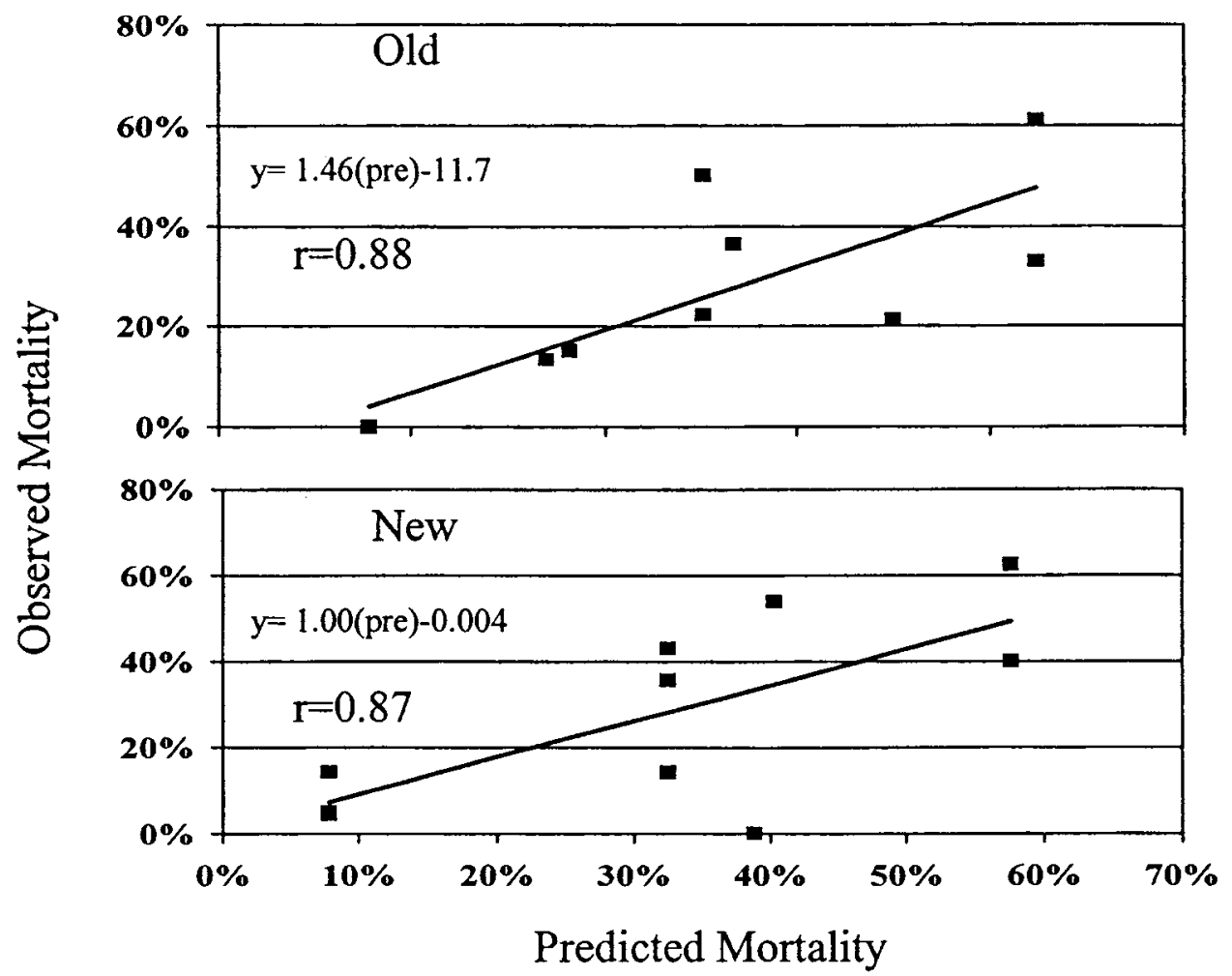

Figure 4. The relation between observed and predicted mortality by using the old (top) and new (bottom) scales. The correlation between observed and predicted values was excellent in the new score $(r=0.87)$, with the slope of the linear regression line close to 1 and a $y$-intercept close to 0 , suggesting accurate predictive ability. In the old score correlation between observed and predicted values was excellent $(r=0.88)$, but the slope of the linear regression line was significantly greater than $1(P<.01)$, and the $y$-intercept was significantly less than $0(P<$ .001). Thus the score overpredicted mortality in patients at low risk (predicted mortality of $<30 \%$ ) and underpredicted mortality in patients with predicted mortalities of greater than $30 \%$.

sode of cardiogenic shock after acute myocardial infarction, and 2 episodes of acute myocarditis) presented with a device after a failed cardiac surgical procedure. The presence of a temporary device is an indicator of the degree of hemodynamic instability and is an important risk factor in the new scale, with a relative risk of 3.3.

Although a previous RVAD emerged as a significant univariate predictor of mortality (relative risk, 3.2; $P=$ $.007)$, the small sample size $(\mathrm{n}=10)$ precluded meaningful analysis. In addition, all 10 patients presented with both a left and right-sided device. Therefore RVAD in situ was excluded as a variable in the new screening scale.

When postcardiotomy shock and previous LVAD insertion were presented to the multivariable model, only previous LVAD insertion emerged as an independent predictor of mortality (OR, 2.83). However, because of the challenges of LVAD insertion in a patient with recent cardiac surgery, we elected to award 2 risk points for postcardiotomy shock in addition to the 2 risk points awarded for a previous LVAD in situ.
Acute myocardial infarction and a diagnosis of ischemic cardiomyopathy were weakly interrelated univariate predictors. Because of the significant overlap between these 2 variables and their failure to emerge as independent predictors in the multivariable analysis, we elected to exclude them in the revised screening scale.

\section{Effect of End-Organ Function}

The most striking finding in the present analysis is the lack of effect of preoperative renal insufficiency. Although measurements of serum creatinine might provide additional information, it is insensitive and lags behind changes in renal function. In addition, interpretation of isolated values is difficult because the context might involve recovery from a remote injury or worsening function from prolonged shock. We have previously found that urine output in the immediate preoperative hours is a sensitive indicator of renal function and was the most important predictor of mortality in the old screening score. However, a more aggressive approach to oliguria and the earlier institution of 
ultrafiltration or hemodialysis has attenuated the effect of this risk factor. As a result, immediate preoperative urine output is no longer represented on the risk factor summation score. However, it is clear from Table 2 that the development of postoperative renal failure is still a strong predictor of outcome after LVAD insertion. Therefore continued attention to the perioperative management of renal function is imperative, despite the fact that it is no longer a preoperative predictor of mortality.

Similarly, the effect of hepatic and right-heart failure has attenuated with time. In the old scale the relative risk of a prolonged prothrombin time ( $>16$ seconds) was 2.4 compared with 2.1 in the new scale. The relative risk of an increased central venous pressure $(>16 \mathrm{~mm} \mathrm{Hg})$, a marker of poor right heart function used to be 3.1 and in the present study has fallen to 2.1. Again, the aggressive treatment of volume overload has decreased the relative effect of both of these risk factors. Earlier institution of continuous venovenous hemodialysis leads to less hepatic congestion and improved right heart function. Decreasing hepatic congestion improves the synthetic function of the liver, thereby improving coagulation and decreasing the need for blood product transfusions. The inflammatory cytokine response to heterologous blood transfusion is associated with worsening right-heart function. ${ }^{18,19}$ Because both increased central venous pressure and prothrombin time were associated with increased risk by means of univariate analysis, they were each awarded 1 point in the revised scoring scale.

\section{Comparison of Screening Scores}

The results of our analysis validate the previous summation score, which was derived from a different cohort of patients from 2 separate institutions. The old score satisfactorily predicted survival, with a threshold of 5 discriminating high-risk patients ( $38 \%$ vs $13 \%, P=.001$ ). The new score provided improved discrimination, with a threshold of 5 representing a $46 \%$ risk compared with the $12 \%$ risk in patients with scores of less than $5(P<.001)$. The area under the ROC curves were similar, with slightly higher accuracy in the new scale (0.77 vs 0.73 ). The relationship between observed and predicted values was greatly improved in the new scale. The old scale overestimated mortality in patients with predicted survival of greater than $70 \%$ and underestimated mortality in high-risk patients with predicted survivals of less than $70 \%$. In contrast, there was excellent correlation between observed and predicted outcomes when the revised scoring scale was used (slope $=$ $1.00, r=0.87$ ).

\section{Summary}

As the effect of mechanical circulatory assistance for the treatment of acute and chronic heart failure increases, a greater proportion of patients will present for device con- sideration at high risk for perioperative mortality. Identification of the preoperative variables, which accurately predict risk, is important for both resource use and for revising management strategies for these challenging patients. A dramatic example of this concept is illustrated in the present report, which documents the changing effect of preoperative renal status on perioperative mortality. Identification of poor urine output in our previous analysis led us to adopt a more aggressive approach to the treatment of impending renal failure. As a result of this paradigm shift, we have improved our perioperative management of overall volume status, and thus poor urine output, increased central venous pressure, and prolonged prothrombin time no longer increase operative risk significantly.

The present analysis suggests that the predominant predictor of risk in contemporary patients receiving LVADs is the preoperative requirement for mechanical ventilation. New strategies aimed at improving pulmonary function might help to mitigate the effect of this variable and improve the clinical results of mechanical circulatory support in an increasingly high-risk patient population. The current study is based on a single center's experience with only one of the available long-term implantable devices. A prospective multicenter experience is needed to validate the conclusions of this report.

\section{References}

1. Sun BC, Catanese KA, Spanier TB, et al. 100 long term implantable left ventricular assist devices: the Columbia Presbyterian Interim Experience. Ann Thorac Surg. 1999;68:688-94.

2. Arabia FA, Smith RG, Rose DS, Arzouman DA, Sethi GK, Copeland JG. Success rates of long-term circulatory assist devices used currently for bridge to transplantation. ASAIO J. 1996;42:542-6.

3. Hunt SA, Frazier OH. Mechanical circulatory support and cardiac transplantation. Circulation. 1998;97:2079-90.

4. Pennington DG, McBride LR, Swartz MT. Implantation technique for the Novacor left ventricular assist system. J Thorac Cardiovasc Surg. 1994;108:604-8.

5. Oz MC, Goldstein DJ, Pepino P, et al. Screening scale predicts patients successfully receiving long-term implantable left ventricular assist devices. Circulation. 1995;92(Suppl):II-169-73.

6. Norman JC, Cooley DA, Igo SR, et al. Prognostic indices for survival during postcardiotomy intra-aortic balloon pumping: methods of scoring and classification, with implications for LVAD utilization. $J$ Thorac Cardiovasc Surg. 1977;74:709-20.

7. Helman DN, Morales DLS, Edwards NM, et al. Left ventricular assist device bridge to transplant network improves survival after failed cardiotomy. Ann Thorac Surg. 1999;68:1187-94.

8. Samuels LE, Kaufman MS, Thomas MP, Holmes EC, Brockman SK, Wechsler AS. Pharmacological criteria for ventricular assist device insertion following postcardiotomy shock: experience with the ABIOMED BVS system. J Card Surg. 1999;14:288-93.

9. Ivanov J, Tu JV, Naylor CD. Ready-made, recalibrated or remodeled? Issues in the use of risk indexes for assessing mortality after coronary artery bypass graft surgery. Circulation. 1999;99:2098-104.

10. Oz MC, Goldstein DJ, Rose EA. Preperitoneal placement of ventricular assist devices: an illustrated stepwise approach. J Card Surg. 1995;10:288-94.

11. American Heart Association. Heart and stroke facts: statistical supplement. Dallas, Tex: American Heart Association; 1998.

12. Rao V, Naka Y, Catanese KA, Flannery MA, Oz MC. Economic costs associated with implantable left ventricular assist device therapy. $J$ Circ Support. 2001;2:31-4. 
13. Tu JV, Jaglal SB, Naylor CD, the Steering Committee of the Provincial Adult Cardiac Care Network of Ontario. Multicenter validation of a risk index for mortality, intensive care unit stay and overall hospital stay after cardiac surgery. Circulation. 1995;91:677-84.

14. Edwards FH, Clark RE, Schwartz M. Coronary artery bypass grafting: the Society of Thoracic Surgeons National Database experience. Ann Thorac Surg. 1994;57:12-9.

15. Hannan EL, Kilburn H, O’Donnell JF, Lukacik G, Shields EP. Adult open heart surgery in New York state: an analysis of risk factors and hospital mortality rates. JAMA. 1990;264:2768-74.

16. Hoy FB, Mueller DK, Geiss DM, et al. Bridge to recovery for postcardiotomy failure: is there still a role for centrifugal pumps? Ann Thorac Surg. 2000;70:1259-63.

17. Muerhcke DD, McCarthy PM, Stewart RW, et al. Extracorporeal membrane oxygenation for postcardiotomy cardiogenic shock. Ann Thorac Surg. 1996;61:684-91.

18. Spanier TB, Oz MC, Levin HR, et al. Activation of coagulation and fibrinolysis in patients with left ventricular assist devices. J Thorac Cardiovasc Surg. 1996;112:1090-7.

19. Goldstein DJ, Seldomridge RA, Chen JM, et al. Use of aprotonin in LVAD recipients reduces blood loss, blood use and perioperative mortality. Ann Thorac Surg. 1995;59:1063-7.

\section{Bound volumes available to subscribers}

Bound volumes of The Journal of Thoracic and Cardiovascular Surgery are available to subscribers (only) for the 2003 issues from the Publisher, at a cost of $\$ 134.00$ for domestic, $\$ 165.85$ for Canadian, and $\$ 155.00$ for international subscribers for Vol 125 (January-June) and Vol 126 (July-December). Shipping charges are included. Each bound volume contains a subject and author index and all advertising is removed. The binding is durable buckram with the Journal name, volume number, and year stamped in gold on the spine. Payment must accompany all orders. Contact Mosby, Subscription Customer Service, 6277 Sea Harbor Dr, Orlando, FL 32887, USA; phone 800-654-2452 or 407-345-4000.

Subscriptions must be in force to qualify. Bound volumes are not available in place of a regular Journal subscription. 